\title{
Ab Initio Study of the Electronic and Phonon Band Structure Of the Mixed Valence Silver Oxide
}

\author{
N. N. Omehe ${ }^{1}$, S. Ehika ${ }^{2}$ \\ ${ }^{1}$ Federal University, Otuoke, Bayelsa State, Nigeria. \\ ${ }^{2}$ Ambrose Alli University, Ekpoma, Edo state, Nigeria.
}

\begin{abstract}
We have performed the electronic band structure and the Phonon dispersion spectra of the mixed valence Silver oxide in the density functional theory (DFT) framework. We used the $L D A+U$ and the HF methods in computing the electronic band structure while the LDA was used for the phonon dispersion calculation. Our structural optimization results agree well with experiment. The $L D A+U$ predicted $A g O$ to be semimetal nature while the HF calculation predicted a semiconductor nature with a fundamental band gap of $1.53 \mathrm{eV}$ which is in good agreement with experiment. In the phonon calculation, we see that the longitudinal optical and the transverse optical split is very small for higher modes corresponding to oxygen. This is the reason while the Raman spectroscopy was not able to observe these modes.

Keywords- Density functional theory, density perturbation functional theory, direct and indirect band gap, electronic band structure, Hartree Fock and local density approximation.
\end{abstract}

\section{Introduction}

Silver oxide $(\mathrm{AgO})$ has attracted so much attention due to its technological applications. It has applications in battery [1], anti-bacterial activity [2], super-resolution near-field structure (RENS) for optical read-write ultra-high density non-volatile memories, in fluorescence imaging and the property of surface enhanced Raman scattering (SERS) in plasmonic devices [3]. It also has applications in optical data storage devices. Silver oxide is a potential candidate in electric-pulse-induced resistive random access memory (RRAM), a technique for the next generation of non-volatile memory [2]. It is a semiconductor whose electronic structure has attracted some controversies, the controversy is about the true state of the mixed oxide. Some studies claim it is silver(I,II) $[4,5,6]$ while some others claim it is silver(I,III) $[7,8]$.

$\mathrm{AgO}$ has been studied experimentally and theoretically to determine its valence mixed state and the electronic band gap. Bielmann et al [5] in their Ultraviolet photoelectron spectroscopy showed that the experimental electronic band gap of $\mathrm{AgO}$ is dependent on oxidation. Their obtained a band gap of $1.09 \mathrm{eV}$ after an immediate oxidation step. On further oxidation, they measured a band gap value of $0.52 \mathrm{eV}$. This band closing is also corroborated in result of the investigation by Raju et al [9]. Park et al [4] in their model of AgO studied by density functional theory using full-potential linearized augmented plane wave (FLAPW) method obtained a small indirect band gap of $0.03 \mathrm{eV}$. Allen et al using the hybrid functional proposed by Heyd et al [10] obtained an indirect band gap of $1.2 \mathrm{eV}$ and a direct band gap of $1.6 \mathrm{eV}$. They quarrelled the model by Park et al, saying it was incapable of modeling the mixed valence silver oxide. The Raman spectroscopy on AgO was also studied by Raju et al [9].

In this study, we investigate the electronic and vibration properties of $\mathrm{AgO}$. To the best of our knowledge, this is the first time a theoretical phonon investigation will be carried out. We apply the HartreeFock (HF) and the $\mathrm{LDA}+\mathrm{U}$ scheme to investigate the electronic band structure of $\mathrm{AgO}$.

\section{Computational Detail}

$\mathrm{AgO}$ crystallizes in a monoclinic structure with a space group $P 121 / \mathrm{c} 1$. The lattice parameters are a $=5.86 \AA, \mathrm{b}=3.48 \AA, \mathrm{c}=5.5 \AA$, and $\beta=107.5^{\circ}$ (taking from ref 11). The lattice contains two nonequivalent $\mathrm{Ag}$ sites: $\mathrm{Ag}(1)$ has two nearest oxygen atoms with the $\mathrm{Ag}(1)-\mathrm{O}$ bond length is $2.18 \AA$; $\mathrm{Ag}(2)$ is surrounded by four oxygen atoms with $\mathrm{Ag}(2)$-O bond lengths of 2.01 and $2.05 \AA$. Fig. 1 show the structure of $\mathrm{AgO}$ adopted for this investigation.

In this study, we have performed structural relaxation, the electronic band structure and Phonon band structure of bulk $\mathrm{AgO}$. In the relaxation of $\mathrm{AgO}$, the starting lattice parameters were adopted from the experimental values of Jansen and Fischer [11]. The two inequivalent Ag occupy the $2 b$ and $2 c$ wyckoff's positions. These two were kept fixed while the position of the oxygen was allowed to evolve. The atomic positions were deemed to have relaxed when the force is $0.01 \mathrm{br} / \mathrm{atom}$. The lattice parameters were then optimized with the relaxed atomic positions. The results of the structure optimization with previous theoretical and experimental results are shown in Table 1. The relaxed coordinate for the oxygen atoms and the $\mathrm{Ag}$ coordinates are given in table II. For the electronic band structure, we made use of the Hartree-Fock (HF) and 
local density approximation LDA+U schemes as implemented in the Abinit package [12, 13]. Projector Augmented Wave (PAW) [14] was use for the LDA+U calculations. In all our calculations, the local density approximation (LDA) as parametrized by Perdew and Zunger [15] was used. The pseudopotentials used in the LDA calculations were norm-conserving [16].

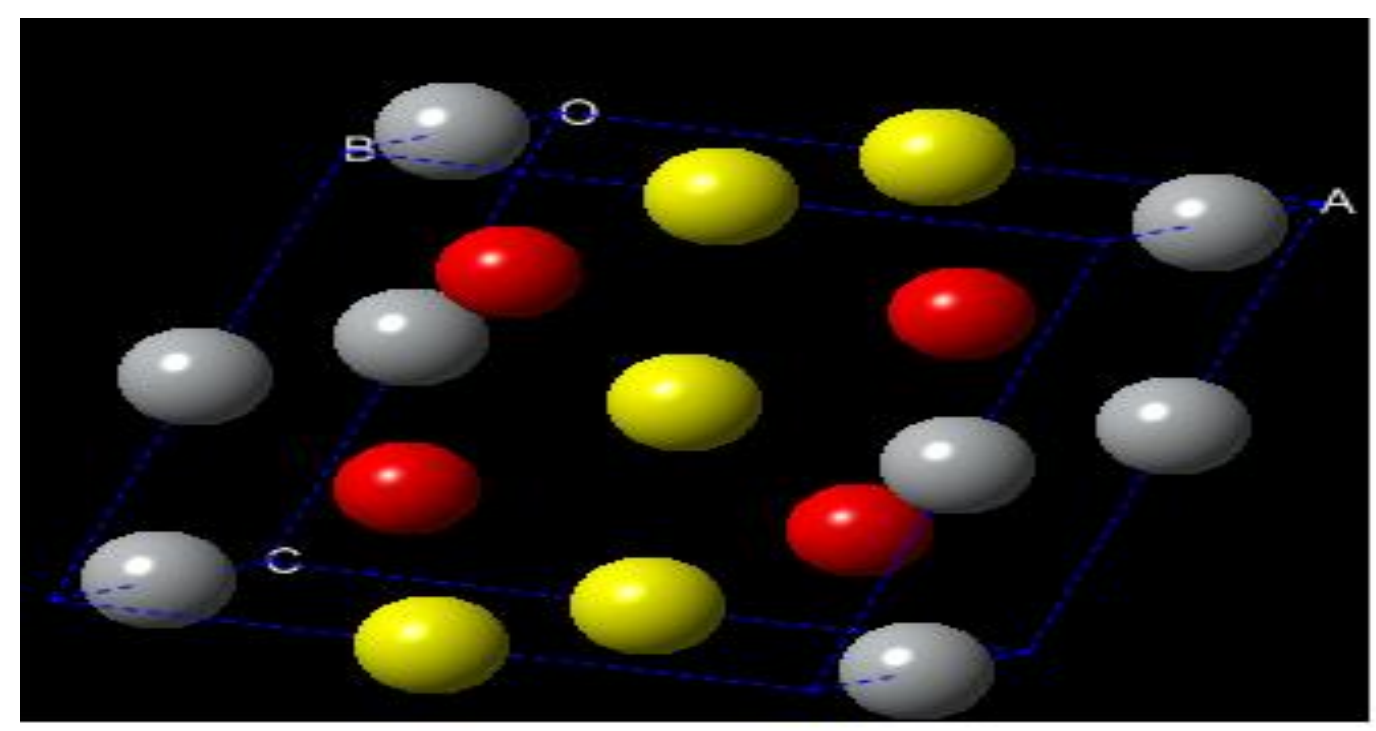

Fig 1. Crystal structure of $\mathrm{AgO}$ from our relaxation. Gray spheres denote linearly coordinated, $\mathrm{Ag}_{\text {lin }}$, yellow spheres denote square planar coordinated $\mathrm{Ag}_{\mathrm{sq}}$, and red oxygen (generated using CrystalMaker).

In all the calculations, the brillouin zone integration for the self-consistency field run was done with a k-point mesh of 128. A kinetic cut-off of 20 hartree with a PAW Energy cut-off for the double grid (allows to transfer data from the normal, coarse, FFT grid to the spherical grid around each atom) of 20 hartree was used in the $\mathrm{LDA}+\mathrm{U}$ calculations. The self-consistency calculation was assumed to have converged when the difference in energy between subsequent iteration was $1.0 \times 10^{-10}$. A plane wave set of 179 was used in the exchange part of the self energy operator; 89 was used for the wave function in self-energy of the HF calculations. The wannier90 [17, 18, 19] package was used in the interpolation of the calculated band structure in the $\mathrm{HF}$ calculation. For the phonon calculations, the kinetic energy cut-off of 30 hartree was used for the generation of the plane waves. The number of wave vectors (q-point) used is 32. Lattice dynamical calculations (phonon dispersion curves and phonon density of states) are performed within the frame work of the self consistent density functional perturbation theory (DFPT) [20, 21, 22].

Table 1. The lattice parameters, bond length and the angle for this work, previous DFT calculations and experiment. The lattice parameters and bond lengths are in Angstrom while angle is in degrees.

\begin{tabular}{|l|l|l|l|l|l|l|}
\hline & This work & Ref 8 & Ref 8 & Ref 8 & $\begin{array}{l}\text { Exp. ref } \\
11\end{array}$ & $\begin{array}{l}\text { Exp ref } \\
23\end{array}$ \\
\hline $\mathrm{a}$ & 5.85 & 5.97 & 4.74 & 5.96 & 5.86 & 5.852 \\
\hline $\mathrm{b}$ & 3.477 & 3.55 & 4.62 & 3.69 & 3.48 & 3.475 \\
\hline $\mathrm{c}$ & 5.499 & 5.57 & 5.6 & 5.53 & 5.5 & 5.495 \\
\hline $\mathrm{Ag}_{\text {lin }}$ & 2.20 & 2.15 & 2.17 & 2.31 & 2.16 & 12.17 \\
\hline $\mathrm{Ag}_{\text {sq }}$ & $2.099,2.099$ & $2.01,2.01$ & 2.17 & $1.99,1.99$ & $2.03,2.02$ & $2.06,2.05$ \\
\hline Angle $\beta$ & $107.7^{\circ}$ & $107.33^{\circ}$ & $90.31^{\circ}$ & $102.85^{\circ}$ & $107.56^{\circ}$ & 107.5 \\
\hline
\end{tabular}




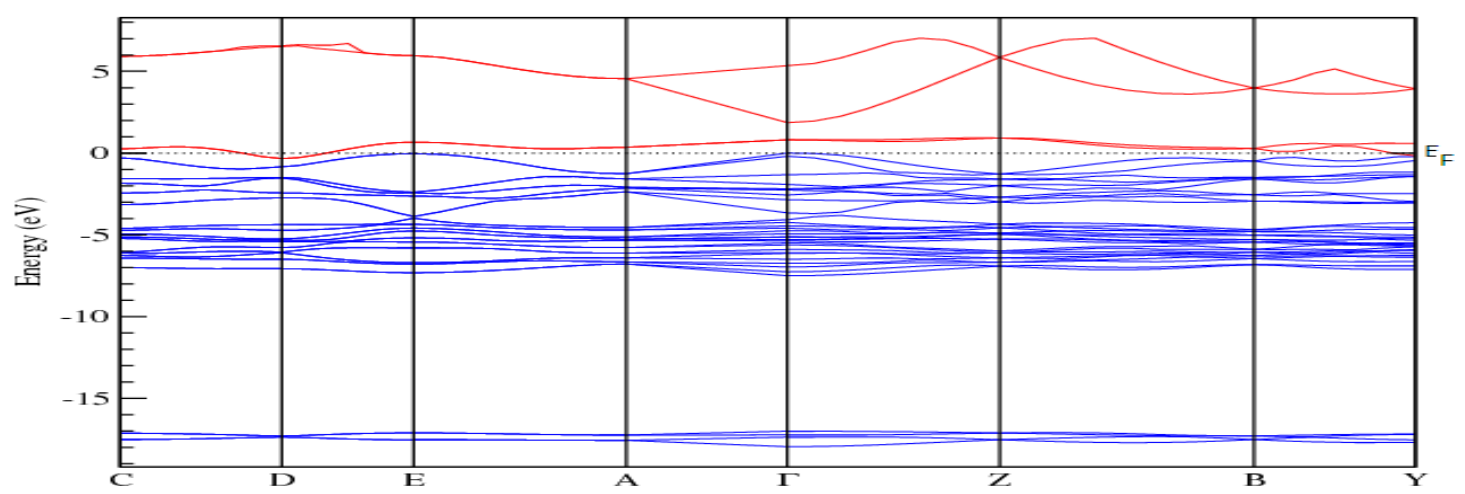

Fig. 2 : The electronic band structure of ago from the LDA+U calculation. The Fermi level is indicated by $E_{F}$.

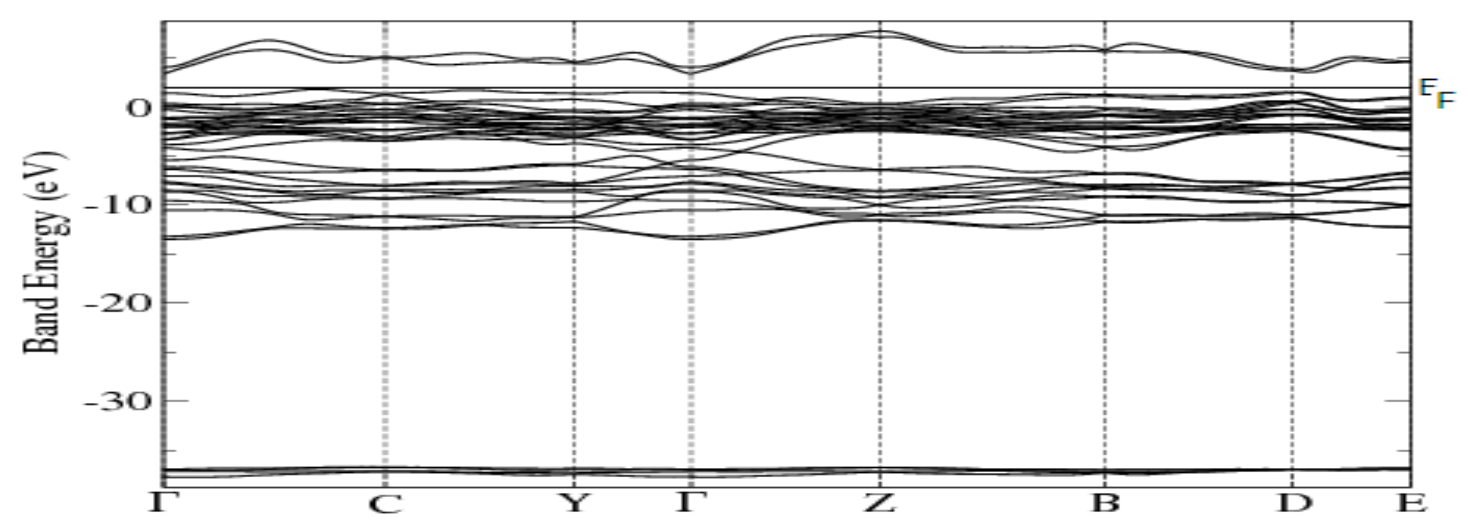

Fig. 3 : The electronic band structure of $\mathrm{AgO}$ from the $\mathrm{HF}$ calculation. The Fermi level is indicated by $\mathrm{E}_{\mathrm{F}}$.

Table 2. The atomic coordinates from the relaxation. There are 8 atoms per unit cell Wyckoff's coordinates (space group number $=14$ )

\begin{tabular}{|l|l|l|l|}
\hline Atom & $x$ & $y$ & $z$ \\
\hline $\mathrm{Ag}_{\mathrm{sq}}$ & 0.5 & 0.5 & 0.5 \\
\hline $\mathrm{Ag}_{\text {lin }}$ & 0.0 & 0.5 & 0.0 \\
\hline $\mathrm{O}$ & 0.264 & 0.85 & 0.23 \\
\hline
\end{tabular}

\section{Results And Discussions}

The electronic band structure of $\mathrm{AgO}$ along lines of high symmetry in the first brillioun zone for the $\mathrm{LDA}+\mathrm{U}$ and $\mathrm{HF}$ calculations are shown in Figs. 2 and 3 respectively. The $\mathrm{LDA}+\mathrm{U}$ calculation predicted $\mathrm{AgO}$ to be a semi-metal. The true semiconductor nature of $\mathrm{AgO}$ was predicted by the $\mathrm{HF}$ calculation with a fundamental indirect band gap of $1.53 \mathrm{eV}$ which is in good agreement with experiment [5, 9] and other theoretical result [8]. The optical direct band gap obtained is $1.85 \mathrm{eV}$. The valence band maximum (VBM) occurred at the $\mathrm{C}$ points while the bottom of the conduction band is located at the $\Gamma$ point. There is however qualitative disagreement with the result of Allen et al. The disagreement is in the location of the valence band maximum (VBM) and the conduction band minimum (CBM). Their VBM is at the $\Gamma$ point while their $\mathrm{CBM}$ is between the $\mathrm{B}$ and $\mathrm{D}$ points. The experimentally observed band gap is $1.1 \mathrm{eV}$.

The total and partial density of states are presented in Fig. 4. From the total DOS in Fig. 4a, it can be seen that the states in the energy window between -0.6 and -0.55 is the $\mathrm{Ag} 5 \mathrm{~s}$ and $\mathrm{O} 2 \mathrm{~s}$ orbital. It is dominated by the $\mathrm{O} 2 \mathrm{~s}$ states. 
(a)
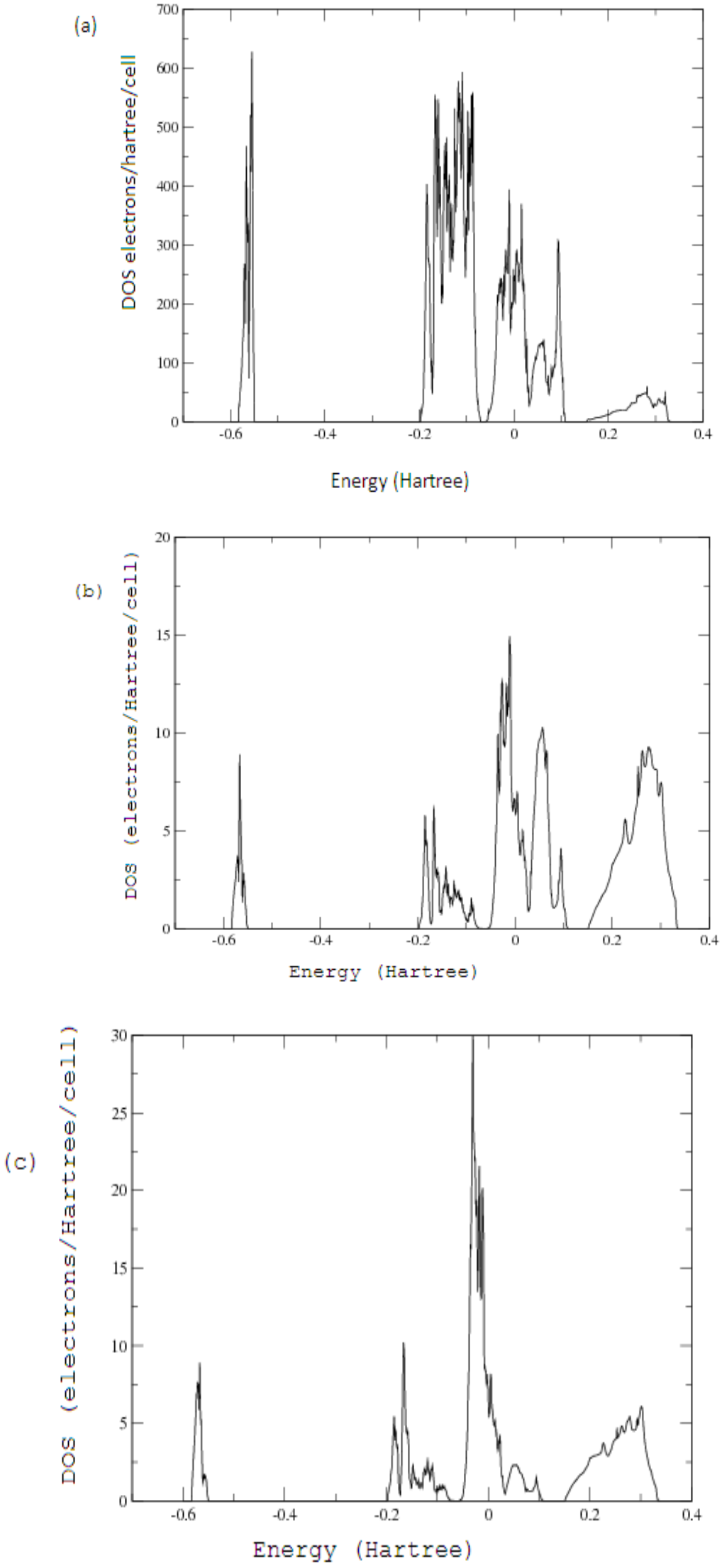


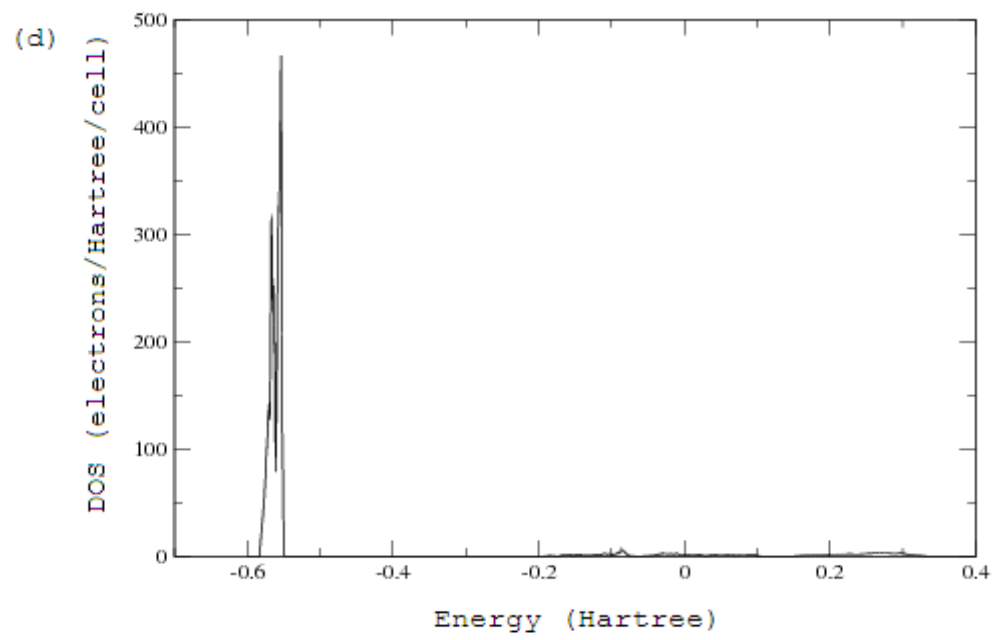

Fig. 4: The density of states (DOS) from LDA+U method. The Fermi energy is at zero. (a) the total DOS (b) partial DOS for $\mathrm{Ag}_{\text {lin }}$ (c) partial DOS for $\mathrm{Ag}_{\text {lin }}$ (d) partial DOS for O.

This is clearly seen from the partial DOS of O in Fig. 4d. Valence band and the bottom of the conduction band are predominantly Ag-d orbitals as seen in the partial density of states of Ag in Figs. 4b and 4c. This Ag-d dominated valence band is in agreement of the result of Allen et al [8].

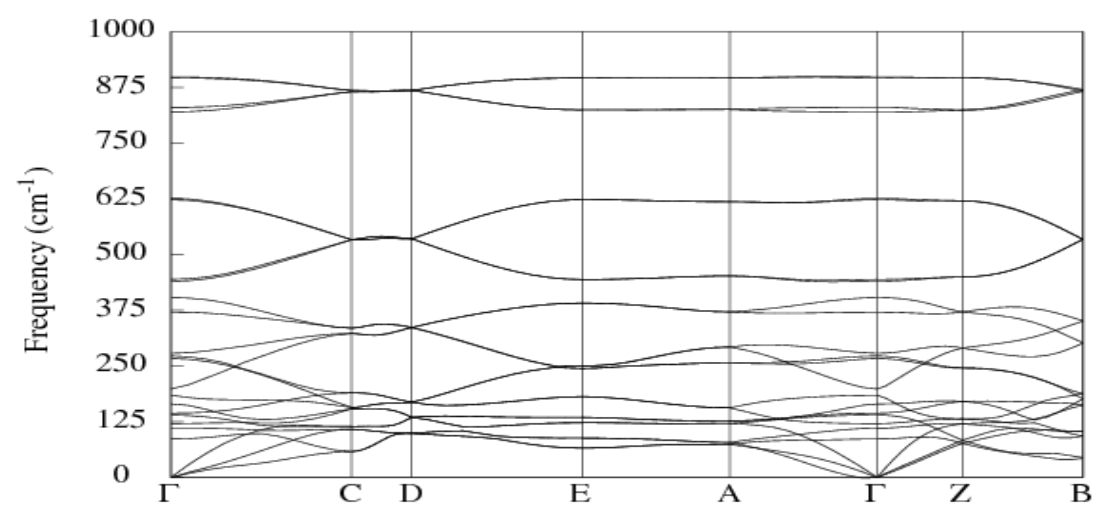

Fig. 5: The Phonon dispersion curve for AgO.

The phonon dispersion curves of bulk $\mathrm{AgO}$ along several high symmetry lines in the brillouin zone are displayed in Fig. 5. There are 8 atoms group into 3 types in the unit cell. This gave a total of 24 frequency modes or bands. Of this number, 3 are acoustic while 21 are optical. The bands are either longitudinal or transverse. The longitudinal acoustic (LA) mode in the direction $\Gamma-\mathrm{C}$ experiences a softening or downward bending to meet the first transverse acoustic mode $\left(\mathrm{TA}_{1}\right)$ at a degenerate point at $\mathrm{C}$. In the $\Gamma-\mathrm{A}$ and $\Gamma-\mathrm{Z}$ directions, the figure reveal an upward bending of (LA). There is a relatively large splitting of the two TA along the $\Gamma$-C and $\Gamma$-A directions when compared to the very small split in the $\Gamma-\mathrm{Z}$ direction. The first three optical modes at $\Gamma$ corresponding to the second square planar coordinated silver $\left(\mathrm{Ag}_{\mathrm{sq}}\right)$ vibrations are found to be 84.7 $\mathrm{cm}^{-1}, 107.7 \mathrm{~cm}^{-1}$ and $119.3 \mathrm{~cm}^{-1}$ representing the longitudinal optical (LO) mode, the second transverse optical $\left(\mathrm{TO}_{2}\right)$ mode and first transverse optical $\left(\mathrm{TO}_{1}\right)$ mode respectively. The six vibration modes or branches belonging to the two linearly coordinated silver atoms were found to be $138.6 \mathrm{~cm}^{-1}, 146.4 \mathrm{~cm}^{-1}, 163.7 \mathrm{~cm}^{-1}$ for $\mathrm{TO}_{1}, \mathrm{~T} \mathrm{O}_{2}$ and LO respectively; and $180.9 \mathrm{~cm}^{-1}, 198.2 \mathrm{~cm}^{-1}$ and $265.5 \mathrm{~cm}^{-1}$ for $\mathrm{TO}_{1}, \mathrm{~T} \mathrm{O}_{2}$ and LO respectively corresponding to the two atoms. The remaining optical branches belong to the four Oxygen atoms. At the Cpoint the two TO modes for the first oxygen atom are degenerate with a frequency of $333 \mathrm{~cm}^{-1}, 271.4 \mathrm{~cm}^{-1}$, $277.0 \mathrm{~cm}^{-1}$ and $369.5 \mathrm{~cm}^{-1}$ corresponds to $\mathrm{TO}_{1}, \mathrm{TO}_{2}$ and $\mathrm{LO}$ respectively. There is a softening of $\mathrm{TO}_{1}$ corresponding to second oxygen atom along the $\Gamma-\mathrm{C}$ direction and becoming degenerate with the LO corresponding to the first oxygen at the C- point. This downward bending can also be seen in $\Gamma-\mathrm{A}$ and $\Gamma-\mathrm{Z}$ directions. It can be clearly seen from the Phonon dispersion curve of Figure 5 that the separation between LO and $\mathrm{TO}_{2}$ branches in the $\Gamma-\mathrm{C}, \Gamma-\mathrm{A}$ and $\Gamma$ - $\mathrm{Z}$ directions is very small. These branches belong to the second oxygen atom. For the third oxygen atom, there is no TO splitting and the branches are not dispersive in the E-A, A- $\Gamma$, $\Gamma-\mathrm{Z}$ directions. The same is observed for the three highest branches which correspond to the fourth oxygen 
atom. The highest mode is at the centre of the $\mathrm{BZ}$ and its value is $894.5 \mathrm{~cm}^{-1}$. The Phonon density of state (PHDOS) for AgO is shown in Figure 5. The PHDOS is divided into three part which are separated by energy gaps. The first part which ranges from 0.0 to $0.0018 \mathrm{Ha}$ is dominated by vibrations of $\mathrm{Ag}$ character while the higher energy vibrations originate dominantly from $\mathrm{O}$ atoms.

Our study of the vibrational properties of $\mathrm{AgO}$ is in disagreement with the Raman spectra of Raju et al [9]. This is because of the very small LO/TO splitting which cannot be resolved experimentally.

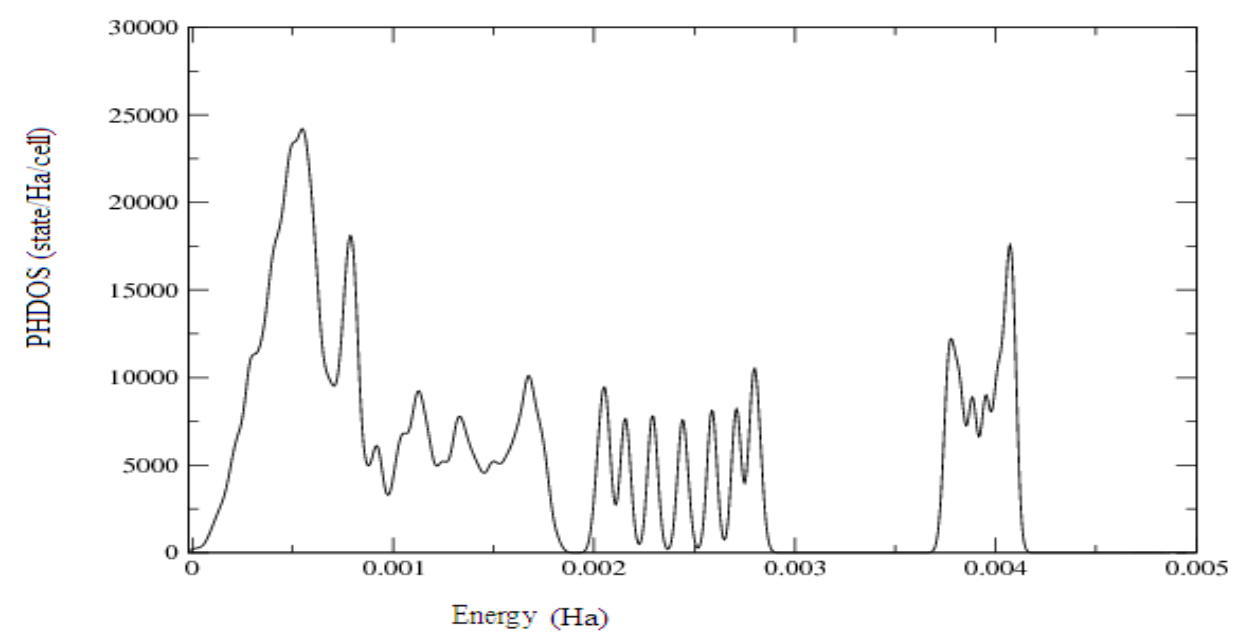

Fig. 6: The Phonon density of state of AgO.

IV.

Conclusions

We have performed the electronic band structure and the Phonon dispersion spectra of the mixed valence Silver oxide in the density functional theory (DFT) framework. We used the LDA+U and the HF methods in computing the electronic band structure while the LDA was used for the phonon dispersion calculation. The LDA+U predicted $\mathrm{AgO}$ to be semimetal nature while the $\mathrm{HF}$ calculation predicted a semiconductor nature with a fundamental band gap of $1.53 \mathrm{eV}$ which is in good agreement with experiment. In the phonon calculation, we see that the longitudinal optical and the transverse optical split is very small for higher modes corresponding to oxygen. This is the reason while the Raman spectroscopy was not able to observe these modes.

\section{References}

[1]. Pan J., Sun Y., Wang Z., Wan P., Liu X., and Fan M., Nano silver oxide (AgO) as a super high charge/discharge rate cathode material for rechargeable alkaline batteries, J. Mater. Chem., 17, 2007, 4820-4825.

[2]. Dong C. Y., Shang D. S., Shi L., Sun J. R., Shen B. G., Zhuge F., Li R. W., and Chen W., Roles of silver oxide in the bipolar resistance switching devices with silver electrode, Appl. Phys. Lett., 98, 2011, 072107-072110.

[3]. Fujimaki M., Awazu K., Tominaga J., and Iwanabe Y., Surface-enhanced Raman scattering from Ag nanoparticles formed by visible laser irradiation of thermally annealed $\mathrm{AgO}_{x}$ thin films, J. Appl. Phys., 100, 2007, 074303-074309.

[4]. Park K., Novikov D. L., Gubanov V. A., and Freeman A. J. Electronic structure of noble-metal monoxides: PdO, PtO, and AgO. Phys. Rev. B 49, 1994, 4425-4431.

[5]. Bielmann M., Schwaller P., Ruffieux P., Gröning O., Schlapbach L., and Gröning P., Ago investigated by photoelectron spectroscopy: Evidence for mixed valence, Phys. Rev. B 65, 2002, 235431-235435.

[6]. Hoflund G. B., Hazos Z. F., and Salaita G. N., Surface characterization study of $\mathrm{Ag}, \mathrm{AgO}$, and $\mathrm{Ag}_{2} \mathrm{O}$ using x-ray photoelectron spectroscopy and electron energy-loss spectroscopy, Phys. Rev. B 62, 2000, 11126-11133.

[7]. Lützenkirchen-Hecht D., and Strehblow H.-H., Anodic silver (II) oxides investigated by combined electrochemistry, ex situ XPS and in situ X-ray absorption spectroscopy Surf. Interface Anal. 41, 2009, 820

[8]. Allen J. P., Scanlon D. O., and Watson G. W. Electronic structure of mixed-valence silver oxides AgO from hybrid densityfunctional theory, Phys. Rev. B 81, 2010, 161103-161107.

[9]. Raju N. R. C, Kumer K. J., and Subrahmanyam A. Physical properties of silver oxide thin film by pulsed laser deposition: effect of oxygen pressure during growth, J. Phys. D 42, 2009, 135411-135417.

[10]. Heyd S., Scuseria G. E. and Ernzerhof M. Hybrid functionals based on a screened coulomb potential, J. Chem. Phys. 118, 2003, 8207-8215.

[11]. Jansen M., and Fischer P., Eine neue darstellungsmethode für monoklines silber(I,III)oxid (AgO), einkristallzüchtung und röntgenstrukturanalyse, J. Less-Common Met. 137, 1988, 123-131.

[12]. Gonze X., Beuken J.-M., Caracas R., Detraux F., Fuchs M., Rignanese G.-M., Sindic L., Verstraete M., Zerah G., Jollet F., Torrent M., Roy A., Mikami M., Ghosez Ph., Raty J.-Y., and Allan D.C., First-principles computation of material properties : the Abinit software project, Computational Materials Science 25, 2002, 478-492. (http://dx.doi.org/10.1016/S0927-0256(02)00325-7)

[13]. Gonze X., Rignanese G.-M., Verstraete M., Beuken J.-M., Pouillon Y., Caracas R., Jollet F., Torrent M., Zerah G., Mikami M., Ghosez Ph., Veithen M., Raty J.-Y., Olevano V., Bruneval F., Reining L., Godby R., Onida G., Hamann D. R., and Allan D. C., A brief Introduction to the Abinit software package. Z. Kristallogr. 220, 2005, 558-562. 
[14]. Blochl P. E. Projector augmented-wave Method, Phys. Rev. B 50, 1994, 17953

[15]. Perdew J. P. and Zunger A., Self-interaction to density functional approximation for many-electron systems, Phys. Rev B 23, 1981, 5048-5079.

[16]. Fuchs M. and Scheffler M. Ab initio pseudopotential for electronic structure calculations of poly-atomic systems using density functional theory, Comput. Phys. Commun. 119, 1999, 67-98.

[17]. Mostofi A. A., Yates J. R., Lee Y.-S., Souza I., Vanderbilt D., and Marzari N., Wannier90: A Tool for Obtaining Maximally Localised Wannier Functions, Comput. Phys. Commun. 178, 2008, 685.

[18]. Marzari N. and Vanderbilt D., Maximally Localized Generalised Wannier Functions for Composite Energy Bands, Phys. Rev. B 56, $1997,12847-12865$

[19]. Souza I., Marzari N. and Vanderbilt D., Maximally Localized Wannier Functions for Entangled Energy Bands, Phys. Rev. B 65, 2001, 035109-035121.

[20]. Gonze X., and Lee C., Dynamical matrices, Born effective charges, dielectric permittivity tensors, and interatomic force constants from density-functional perturbation theory, Phys. Rev. B 55, 1997, 10355-10368.

[21]. Lee C., and Gonze X., Ab initio calculation of the thermodynamic properties and atomic temperature factors of $\mathrm{SiO}_{2} \alpha$-quartz and stishovite, Phys. Rev. B 51, 1995, 8610-8613.

[22]. Baroni S., Gironcoli S, Corso A., and Giannozzi P., Phonons and related crystal properties from density-functional perturbation theory, Rev. Mod. Phys. 73, 2001, 515-562. 